\title{
Cloud edge computing in the loT
}

\author{
Ilhem Fajjari ${ }^{1} \cdot$ Fouad Tobagi $^{2} \cdot$ Yutaka Takahashi $^{3}$ \\ (C) Institut Mines-Télécom and Springer International Publishing AG, part of Springer Nature 2018
}

The focus of this special issue is on the Internet of Things (IoT) with a particular emphasis on the use of Cloud edge computing as a building block of the IoT architecture and a key infrastructural support for IoT applications.

Cloud edge computing provides a number of benefits including enhanced network performances, lower operational costs, alleviated network congestion, and improved survivability. However, it raises new challenges in terms of device deployment, application monitoring, resources management, etc. The objective of this special issue is to capture the latest advances in this research field and merging standards.

Nine papers have been accepted for this special issue. Reviewers' comments were very helpful to select the most significant contributions as well as to improve the content, quality, and presentation of the accepted papers. These papers address some of the most challenging new problems. They provide an overview of and in-depth research, development, and deployment efforts on the Cloudification of IoT, specifically on the design of fog infrastructure for IoT, fog resource optimization for IoT applications, and security and privacy enhancements in cloud fog environments. Hereafter, a summary of each paper accepted in this special issue is described.

\section{Security and privacy}

- Paul Fremantle and Benjamin Aziz in their paper entitled "Cloud-based Federated Identity for the Internet of Things" put forward a system enabling the federation of various parties including the manufacturer, the identity

Ilhem Fajjari

ilhem.fajjari@orange.com

Orange Labs, Châtillon, France

2 Stanford University, Stanford, CA, USA

3 Kyoto University, Kyoto, Japan provider, and the device identity management. Such a system relies on an extended version OAuth2 to provide device and user registration processes. To prove the feasibility of their proposal, the authors introduce a prototype while describing its performances in terms of energy usage, cost, and capacity metrics.

- Usman Shafique, Abid Khan, Abdur Rehman, Faisal Bashir, and Masoom Alam in their paper entitled "Detection of Rank Attack in Routing Protocol For Low Power and Lossy Networks" address the detection of malicious nodes in wireless sensor nodes deployed for IoT applications such as smart building and smart health. The authors make use of the Low power and Lossy Network Routing Protocol based on IPv6. In doing so, the malicious node can easily update the rank and hence be selected during the routing process. The authors propose a new protocol denoted SBIDS which makes use of the freshness of control message.

\section{Practical systems and platforms}

- Daniele Mazzei, Giacomo Baldi, Gabriele Montelisciani, and Gualtieroi Fantoni in their paper entitled "A Full Stack for Quick Prototyping of IoT Solutions" put forward a new solution aiming to speed up the prototyping process of IoT solution. To do so, they define technical specifications and design requirements for a speed prototyping of IoT for horizontal stack design. The technical specifications deal with device hardware, software, and network layer. Moreover, an implementation of a cloud device manager is proposed, in addition to a data collection and storage engine for IoT data. Finally, a full stack component solution for embedded devices is proposed, Zerynth Stack. This later is instantiated for the implementation of the ROI (Refrigeration On Internet) which represents a smart product in real-world scenario. The solution is successfully applied to a real industrial application. 
- Giuseppe Baruffa, Mauro Femminella, Matteo Pergolesi, and Gianluca Reali in their paper entitled "A Big Data Architecture for Spectrum Monitoring in Cognitive Radio Applications" deal with the cognitive radio networks in the context of IoT. Specifically, they address the problem of the spectrum sensing in cognitive radio in the Cloud computing infrastructure. To do so, the authors introduce a monitoring platform capable of providing the spectrum sensing as a service in order to cope with the limitations of continuous sensing task.

\section{Resources optimization}

- Nitinder Mohan and Jussi Kangasharju in their paper entitled "Placing It Right!: Optimizing Energy, Processing and Transport in Edge-Fog Clouds" design a new utility infrastructure, namely Edge-Fog Cloud. Their main idea is to merge fog and edge computing infrastructures. Hence, computing resources are made available at the edge and fog infrastructures while the storage of already computed data is kept in the centralized cloud. Besides, two new task placement algorithms are proposed where the objective consists in matching the jobs and available resources.

- Hafizur Rahman, Nurzaman Ahmed, and Md. Iftekhar Hussain in their paper entitled "A QoS-aware hybrid data aggregation scheme for Internet of Things" deal with the data aggregation problematic for wireless sensor networks in the context of IoT. Authors propose a hybrid Quality of service-Aware Data Aggregation (QADA) scheme, which combines cluster and tree-based approaches in order to reduce network power consumption, traffic load, and increase bandwidth utilization.

\section{Novel models, architectures, and protocols}

- Vikas S. Shah in his paper entitled "Multi-Agent Cognitive Architecture enabled IoT Applications of Mobile Edge Computing" contributes to establish an empirical multi-agent cognitive architecture framework for continuous transition of IoT APIs. To do so, a cognitive agent-oriented approach is proposed to proactively subordinate IoT APIs with heterogeneous cloud. The approach introduces the rationalization of cognitive agent creation, migration, and control. The proposed solution achieves transparency in the heterogeneity and distribution of IoT APIs. The paper also illustrates the architecture impact analysis with the indicative capabilities of autoscaling, predictability in heterogeneous cloud resource use, responsiveness and attainment of IoT APIs.

- Nurzaman Ahmed, Hafizur Rahman, and Md. Iftekhar Hussain in their paper entitled "An IEEE 802.11ah-based scalable network architecture for Internet of Things" deal with the optimization of resource usage in dynamic smart city networks. To do so, they address the limitations of IEEE 802.ah and propose some adjustments to this standard in order to optimize the dynamic resource allocation for smart city networks. In addition to enhancing the communication distance among low-power devices, the proposed IEEE 802.11ah-based relay solution aims to handle the heterogeneous traffic pattern and the dynamic aspect of multi-hop smart city networks.

Basically, they proposed two main enhancements for IEEE 802.11ah standard: (i) extending connectivity by incorporating a relay access point and (ii) supporting node dynamicity and traffic heterogeneity by proposing a proper channel access scheme.

- Mauro Femminella, Matteo Pergolesi and Gianluca Reali in their paper entitled "IoT, Big Data and Cloud Computing Value Chain: Pricing Issues and Solutions" address the problem of service pricing for IoT services in Cloud platforms. To do so, the authors motivate the problem and explain the $5 \mathrm{~V}$ requirements: Volume, Velocity, Variety, Veracity, and Value and expose the related pricing models found in the literature. Besides, they introduce a reference architecture for IoT applications based on big data management in order to propose a pricing strategy. In this context, authors put forward a mapping of the needed infrastructure in their proposed model. They quantify the amount of resources required to update a batch view with new data, and the size of the batch views.

Acknowledgments The guest editors would like to thank the authors who contributed with their valuable work to this special issue as well as to the reviewers for their timely reviews of the submitted articles. The guest editors would like to express their deep appreciation to Springer Annals of telecommunications Editor-in-Chief, Guy Pujolle, for giving this opportunity as well as the journal editorial staff for their continuous support and guidelines during the process of this publication. 